INPLASY

PROTOCOL

To cite: Yang et al. The impact of platinum-containing chemotherapies in advanced triple-negative breast cancer: meta-analytical approach to evaluating its efficacy and safety. Inplasy protocol 202110070. doi: 10.37766/inplasy2021.1.0070

Received: 19 January 2021

Published: 19 January 2021

Corresponding author: Sheng Liu

yrshtcm@163.com

Author Affiliation: Institute of Chinese Traditional Surgery, Longhua Hospital, Shanghai University of Traditional Chinese Medicine

Support: National Natural Science Found.

Review Stage at time of this submission: Data analysis.

Conflicts of interest:

None.

\section{The impact of platinum-containing chemotherapies in advanced triple-negative breast cancer: meta-analytical approach to evaluating its efficacy and safety}

Yang, R1; Shi, YY2; Han, XH³; Liu, L44.

Review question / Objective: (1) participants: patients received a definitive diagnosis of advanced TNBC by pathology or cytology; (2) interventions and comparisons: intervention groups received platinum-containing chemotherapy and control groups received either nonplatinum-containing or platinum-containing chemotherapy combined with target drugs. Whether the blind method was not limit. Basic conditions between the two groups were similar and comparable; (3) outcomes: PFS, OS, ORR, and side effects were evaluated in this study.

Condition being studied: Advanced triple-negative breast cancer.

INPLASY registration number: This protocol was registered with the International Platform of Registered Systematic Review and Meta-Analysis Protocols (INPLASY) on 19 January 2021 and was last updated on 19 January 2021 (registration number INPLASY202110070).

\section{INTRODUCTION}

Review question / Objective: (1) participants: patients received a definitive diagnosis of advanced TNBC by pathology or cytology; (2) interventions and comparisons: intervention groups received platinum-containing chemotherapy and control groups received either nonplatinum-containing or platinum-containing chemotherapy combined with target drugs. Whether the blind method was not limit. Basic conditions between the two groups were similar and comparable; 
outcomes: PFS, OS, ORR, and side effects were evaluated in this study.

Condition being studied: Advanced triplenegative breast cancer.

\section{METHODS}

Participant or population: Patients received a definitive diagnosis of advanced TNBC by pathology or cytology.

Intervention: platinum-containing chemotherapies in advanced triplenegative breast cancer.

Comparator: Non-platinum-containing chemotherapies in advanced triplenegative breast cancer

Study designs to be included: Published randomized controlled clinical studies of platinum-containing chemotherapies for advanced TNBC before November.

Eligibility criteria: To collect published randomized controlled clinical studies of platinum-containing chemotherapies for advanced TNBC before November 2020.

Information sources: PubMed, Medline, Embase, Clinicaltrials.gov, Cochrane Library, CNKI, CBM, and the Chinese Cochrane Center.

Main outcome(s): Progression-free survival (PFS), overall survival (OS), objective response rate (ORR), and side effects were evaluated in this study.

Quality assessment / Risk of bias analysis: (1) random sequence generation (selection bias); (2) allocation concealment (selection bias); (3) blinding of participants, personnel, and outcome assessment (performance bias and detection bias); (4) incomplete outcome data (attrition bias); (5) selective reporting data (reporting bias).

Strategy of data synthesis: Review Manager v5.3 was used for data analysis. The 12 test was used for quantitative analysis of interstudy statistical heterogeneity. Generally, an $12>50 \%$ indicates substantial heterogeneity, and thus, a random-effect model should be used. When there is no evidence of statistical heterogeneity among studies (12 $<50 \%$ ), a fixed-effect model should be used for the meta-analysis.

Subgroup analysis: For dichotomous outcomes (adverse events).

Sensibility analysis: None.

Country(ies) involved: China.

Keywords: Advanced triple-negative breast cancer, Met as tat ic, P latinum, Chemotherapy, Meta-analysis.

Contributions of each author:

Author 1 - Rui Yang.

Author 2 - You-yang Shi.

Author 3 - Xiang-hui Han.

Author 4 - Sheng Liu. 